2017-11-30

\title{
Extending Motion Detection to Track Stopped Objects in Visual Multi-Target Tracking
}

Jacob H. White

Brigham Young University, snowflakeobsidian3.14@gmail.com

Karl T. Salva

Air Force Research Laboratory Sensors Directorate, kicksalva@gmail.com

Randal W. Beard

Brigham Young University, beard@byu.edu

Follow this and additional works at: https://scholarsarchive.byu.edu/studentpub

Part of the Electrical and Computer Engineering Commons

\section{BYU ScholarsArchive Citation}

White, Jacob H.; Salva, Karl T.; and Beard, Randal W., "Extending Motion Detection to Track Stopped Objects in Visual Multi-Target Tracking" (2017). All Student Publications. 219.

https://scholarsarchive.byu.edu/studentpub/219 


\title{
Extending Motion Detection to Track Stopped Objects in Visual Multi-Target Tracking
}

\author{
Jacob H. White ${ }^{1}$, Karl T. Salva ${ }^{2}$, Randal W. Beard ${ }^{1}$
}

\begin{abstract}
Various solutions to visual multi-target tracking have been proposed, but many of them are not capable of running in real time from a moving camera on an unmanned aerial vehicle (UAV). We present a tracker that runs in real time and tracks multiple objects while accounting for camera motion on a UAV. Our algorithm is capable of processing over 10 frames per second on a 1280x720 video sequence.

We utilize Recursive-RANSAC, an efficient algorithm for tracking multiple objects in clutter. Our work combines motion detection with optical flow and feature matching to allow stationary objects to be tracked. We use a feature prioritization algorithm to reduce computational complexity and spatial redundancy. We also present a ghost track reduction method which prevents tracking non-existent objects when true objects are no longer visible. We demonstrate the performance of our tracker on a moving camera video sequence. Video results are available at https://youtu.be/6bXjKb_-6qY.
\end{abstract}

\section{INTRODUCTION}

Visual multi-target tracking from an unmanned aerial vehicle (UAV) has many applications including wide area surveillance, search and rescue, photography, law enforcement, and military operations. These applications, however, have different requirements than those of traditional state-ofthe-art trackers. A tracker in these scenarios must be able to run in real time from a UAV, track from a moving camera, and track multiple objects simultaneously.

Perhaps the most critical distinction between our work and state-of-the-art trackers is the requirement that the algorithm not only run in real-time but also run on-board the UAV. Onboard computation avoids many of the challenges inherent in point-to-point video links including data corruption and compression artifacts, which can degrade the performance of the tracker. Tracking on-board the UAV often limits the available computational power.

Many state-of-the-art algorithms commonly used on stationary cameras, such as image-based background subtraction, cannot be used on a moving camera, because the pixel locations of stationary objects are not constant across time.

There are many single object trackers [1], [2], [3], [4], some of which are capable of running in real time. However, it is difficult to use these trackers for multiple objects because they lack automatic track initialization and, assuming the

*This work has been funded by the Center for Unmanned Aircraft Systems (C-UAS), a National Science Foundation Industry/University Cooperative Research Center (I/UCRC) under NSF award Numbers IIP1161036 and CNS-1650547, along with significant contributions from CUAS industry members.

${ }^{1}$ J. H. White and R. W. Beard are with the Department of Electrical and Computer Engineering, Brigham Young University, Provo, UT 84602, USA.

${ }^{2}$ K. T. Salva is with the Sensors Directorate, Air Force Research Laboratory, 1864 4th St, Wright-Patterson AFB, OH 45433, USA. required computation scales linearly, may no longer run in real time when tracking multiple objects.

Multiple object trackers [5], on the other hand, can automatically initialize new tracks. These trackers rely on a multiple-class object detector trained using Deep Learning. There are two main obstacles to using object detectors for tracking from a UAV. First, many of these object detectors do not run in real time. For example, DPM [6] and Faster R-CNN [7], two commonly used object detectors in the Multiple Object Tracking Challenge [8], run at 0.5 and 5 frames per second respectively. Recently several real-time object detection frameworks have been proposed, including YOLO [9] and SSD [10]. These may enable object detectors to be used for real time tracking. Even so, real-time performance is only achieved with a GPU, which may not be feasible to carry on-board a small UAV. Second, object detectors struggle to detect far away objects typical in aerial imagery, likely because they are not usually trained on aerial imagery. Retraining the object detectors on aerial imagery can improve performance [11].

Wide-Area Motion Imagery (WAMI) trackers [12], [13] must use a different set of algorithms because of the enormous camera resolution and limited frame rate of 1 frame every 1 or 2 seconds. WAMI trackers are one of the few trackers that rely on motion detection rather than a multiclass object detector or a user-specified bounding box to initialize tracks. However, the large image resolution makes it very challenging to process in real time. As a result, we focus in this paper on more standard resolution imagery taken at lower altitudes.

The contribution of this paper is a multi-target tracker capable of tracking moving and stopped objects in real time from a moving camera. Our work uses Recursive-RANSAC [14] for track estimation and track management. Our work extends the tracker presented in [15] which successfully tracks multiple objects from a moving camera but fails to track these objects once they have stopped. To track stopped objects, we combine the motion detector used in [15] with BRIEF feature matching [16] and Lucas-Kanade optical flow [17] for persistent point tracking. We use grid-based feature prioritization, denoted as GFP, to keep the number of tracked points small enough to run in real time. We use a track failure detection method to reduce non-existent "ghost" tracks that sometimes arise during track occlusions.

The remainder of the paper is outlined as follows. Sections 2 and 3 describe motion detection and Recursive-RANSAC, the previous methods we extend in this paper. Section 4 and 5 describe our method of persistent point tracking and reducing 

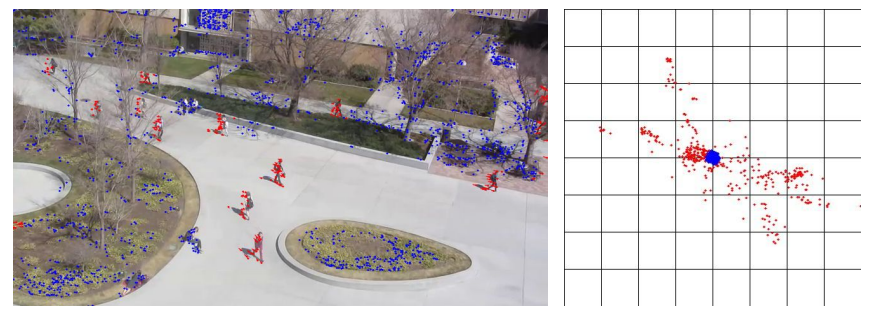

Fig. 1. Motion detection results. Each point position (left) and its corresponding net velocity (right) are plotted. Points with a net velocity above a certain threshold ( 0.2 pixels in this case) are classified as moving points (red), while points with a velocity below this threshold are classified as non-moving points (blue). The grid spacing of the velocity graph (right) represents 1 pixel. Note that Lucas-Kanade optical flow gives sub-pixel accuracy and thus allows pixel movements as small as 0.2 pixels to be detected with very few false positives.

duplicate points using grid-based feature prioritization. Section 6 describes a method for detecting tracking failure. Section 7 summarizes the complete visual multi-target tracker. The results on a moving camera video sequence are discussed in Section 8.

\section{KLT-Based Motion Detection Using HOMOGRAPHY OUTLIERS}

A simple motion detection technique that works on a moving camera is using homography outliers as described in [15]. Good Features to Track [18] are first detected in the current frame and then matched to the previous frame using optical flow. A homography is then fit to these points to describe the motion between the previous and current frames. Outliers to the homography are classified as moving points (see Algorithm 1). The results of this algorithm on a moving camera sequence are shown in Figure 1 .

The homography works well to align the frames if the motion is mostly rotational or if the UAV altitude is large when compared to the non-planar depth variations. This is often true for UAVs. When these assumptions do not hold, stationary objects can appear to be moving. This effect is known as parallax. There are more complicated methods for motion detection that account for parallax [19], [20], [21].

\begin{tabular}{l}
\hline Algorithm 1 Motion Detection \\
\hline 1: Detect features in the current frame \\
2: Calculate velocity of features using LK Optical Flow \\
3: Align previous and current frame using a homography \\
4: Transform points in previous frame to current frame \\
5: Subtract camera motion to obtain net pixel velocity \\
6: Threshold net pixel velocity to find moving points \\
\hline
\end{tabular}

\section{RECURSIVE-RANSAC}

Moving points found using motion detection are then fed into Recursive-RANSAC, a newly proposed algorithm for multi-target tracking presented in [14]. At each time step Recursive-RANSAC searches for new models using RANSAC. When a sufficient number of inliers are detected, a new track is initialized. Existing tracks are propagated forward using a Kalman Filter. Probabilistic Data Association [22] is used to account for measurement association uncertainty. Each track is given an inlier score based on the percentage of time steps in which the track is detected. Recursive-RANSAC also has a track management system that merges similar tracks and removes tracks that have an inlier score lower than the minimum threshold.

\section{Persistent Point Tracking Using Optical FLOW AND FEATURE MATCHING}

Using motion detection as an input to Recursive-RANSAC allows any moving object to be tracked. However, moving objects that subsequently stop can no longer be tracked using this approach. For this reason, we propose extending motion detection to track stopped objects by using long-term point tracking. Long-term point tracking can be done using Optical Flow or Feature Matching. Only one method is required, but combining both has the potential to improve overall tracking accuracy. In this paper we will use Lucas-Kanade Optical Flow [17] and BRIEF Feature Matching [16]. Though there are more sophisticated feature descriptors, BRIEF has the advantage of being easily run in real time, while still being accurate.

Each point being tracked will be denoted as the tuple $\phi_{t, i}^{s}=\{s, p, v, a, f\}$, where $t$ is the time step, $i$ is the point index, $s$ is the source index, $p$ is the point position, $v$ is the point net velocity, $a$ is the point track association, and $f$ is the feature descriptor. The net velocity for persistent points is calculated in the same manner as described in the motion detection algorithm. The track association is determined by R-RANSAC when a new track is initialized or when points fall within the gate of an existing track. The feature descriptor is stored the first time the tracked point is initialized. This collection of information is sufficient to allow long-term point tracking using optical flow and feature matching.

Optical flow calculations are made between consecutive frames (in other words the template is updated each frame). This method of point tracking is potentially more susceptible to gradual drift, but is more accurate between consecutive frames because it avoids jerky estimates due to non-translational image warps and non-rigid object motion. Feature matching, on the other hand, is calculated without updating the template at each time step. This allows lost points to be recovered and has the potential to help distinguish between crossing and interacting targets (See Figure 2). Features are only matched if the feature hamming distance falls below a set threshold. The location of the associated track (assigned by Recursive-RANSAC) defines a search region which reduces unnecessary feature comparisons.

\section{Grid-BAsed Feature Prioritization (GFP)}

If every moving feature were stored in memory and tracked using both optical flow and feature matching, the large number of accumulating features would quickly become computationally intractable (see Figure 3. As a result, features associated with a particular track must be prioritized and less-important features thrown out. To prioritize these 


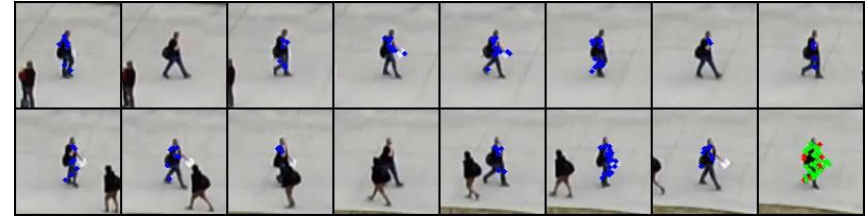

Fig. 2. Feature matches over time. Features are detected in the current frame (bottom right, shown in green) and successfully matched to previous frames (matches shown in blue). Few (in this case none) of the features on the original target are matched to the crossing target. Note that the number of matched features in each frame grows and shrinks over time as the appearance of the target changes.

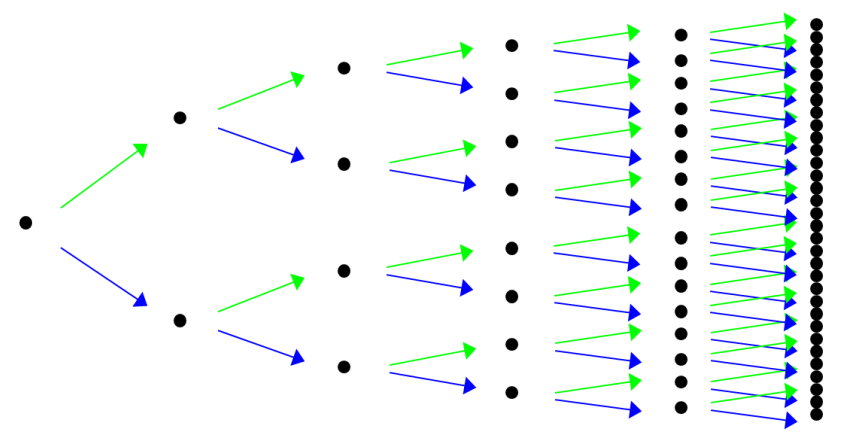

Fig. 3. Each new detected point is tracked using both optical flow (green) and feature matching (blue). Without a method that filters out old or redundant points, the resulting "explosion" of points becomes computationally intractable.

features we use something we will call grid-based feature prioritization (GFP), based on the feature prioritization algorithm in OpenCV's GoodFeaturesToTrack function [23].

GFP adds incoming features to a temporary grid data structure, while ensuring that none of the features are closer than the minimum allowed distance between features. Before being added to the grid the features are sorted by priority. Initially the grid is empty and so almost all of the highpriority features are added. But as more and more features are added, eventually the lower-priority features no longer meet the minimum distance requirement and so they are discarded (Algorithm 2). The grid data structure is used for efficiency so that whenever a new feature is added only the adjacent cells need to be searched rather than searching the entire grid (see Figure 4).

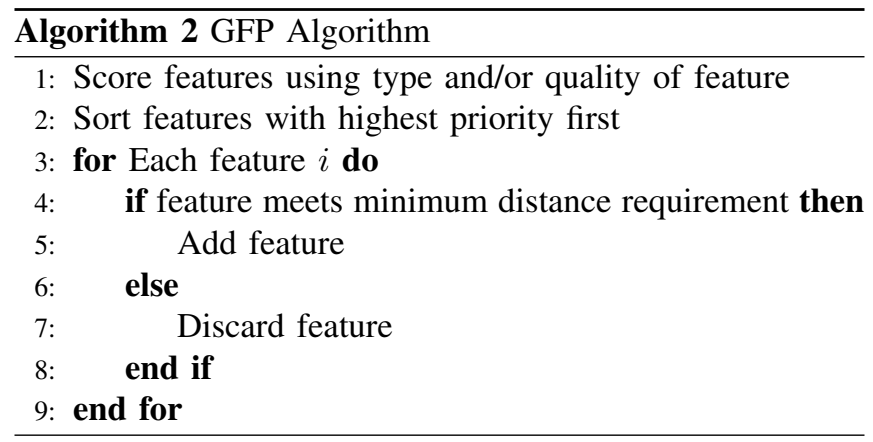

The main advantage of this strategy is that it maximizes the amount of information gained from each area of the im-

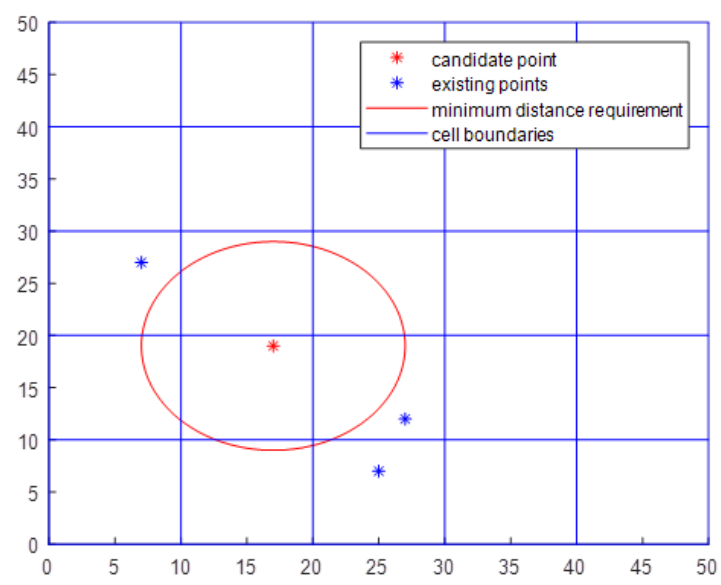

Fig. 4. The GFP algorithm can be optimized by storing the points in a grid data structure. As a result of this modification, only the adjacent cells ( 9 cells total) must be searched each time a new candidate point is added, as opposed to searching the entire collection of points.

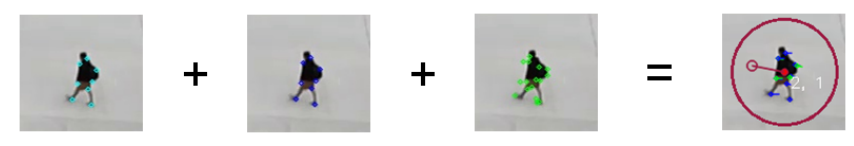

Fig. 5. Moving object detections (teal), feature matches (blue), and optical flow (green) are combined using grid-based feature prioritization (GFP).

age while reducing computational complexity. For example, when optical flow is used to track non-rigid objects such as pedestrians, the features quickly clump together because of crossing limbs. After some time the points are basically duplicates of one another and the extra computation no longer provides any additional information to the tracker. The minimum distance requirement ensures that each point is unique and contributes a valuable piece of information to the tracker.

In our setup, we have chosen the moving object detection to be the highest priority because it is relied on for track initialization and gives original information about the location of new and existing targets. Feature matching and optical flow are not original sources since they can only be used to compare the current frame to earlier frames in which the moving objects were detected. Feature matching and optical flow are used whenever the corresponding region of the image has no moving object detections. The result of the feature prioritization algorithm is shown in Figure 5.

\section{Reducing GHOSt TRACKS}

Often non-existent ghost tracks are a side-effect of attempting to track stationary objects. Ghost tracks usually occur when an object being tracked disappears behind an occlusion. If the tracking methods do not detect that the object is no longer visible they will begin to track the occlusion instead of the original object. Examples of ghost tracks are shown in Figure 6.

Automatic detection of tracking failure is arguably just 

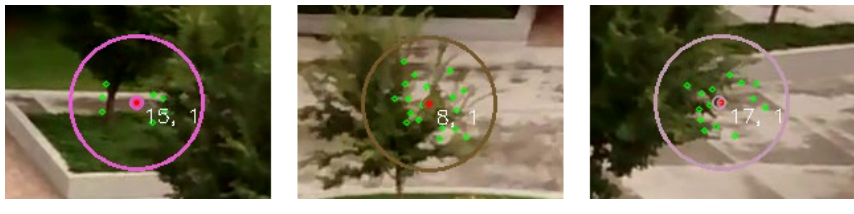

Fig. 6. Examples of non-existent "ghost" tracks.

as important as automatic track initialization. In this paper we detect track failure by comparing the current frame to a background image to determine whether the current frame still contains the object. If the two images are different, the difference implies that something is present in the current frame. If the current frame is identical to the background image, the object being tracked must no longer be visible. Optical flow can be used to determine similarity between the background image and the current frame. If the calculated motion is greater than a set threshold, the point is kept, otherwise it is discarded to avoid creating ghost tracks. In our tests a threshold of 1 pixel gave good results.

Instead of maintaining a background model, which on a moving camera is not trivial, we look for a recent previous image that contains background near the object of interest. The main challenge is to decide which frame contains background. A simple method to determine whether an area of the image contains foreground or background is to keep a history of recent measurements and check for measurements in the area of interest at the given time step. If there are no nearby measurements at that time step, the frame is likely to contain only background. Since not all movement is observed in the image, there is still some uncertainty in whether these frames contain background. To improve the ghost track reduction accuracy, multiple candidate background frames are compared to the current frame. After a few time steps the tracked point is flagged as a verified stationary point and no further comparisons are performed.

On a stationary camera, optical flow can be calculated directly on the two images without any image registration. However, on a moving camera the images must first be aligned using a homography or other transformation. In our approach we do not calculate this homography from scratch but instead concatenate previously-calculated homographies to determine the transformation. This is more susceptible to drift over time, but since most of the time we are only going back a couple of frames the registration is quite accurate.

Other methods for detecting track failure include forwardbackward error [24] and affine motion dissimilarity [18]. Analyzing and comparing these track failure detection methods is beyond the scope of this paper.

\section{ViI. Complete Tracker Pipeline}

The complete visual multi-target tracker pipeline is shown in Figure 7. The diagram shows the flow of tracked points through the system. Three measurement sources are shown on the left of the figure: KLT-based motion detection, KLT point tracking, and BRIEF feature matching. These sources are combined using Grid Feature Prioritization (GFP) to

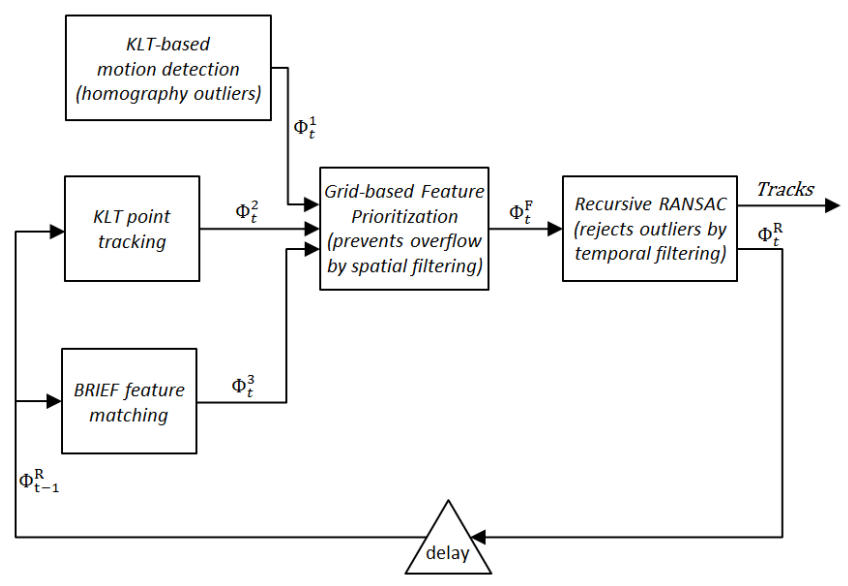

Fig. 7. Block diagram of the complete visual multi-target tracker

eliminate redundant measurements. The points are then fed into Recursive-RANSAC to remove outlier measurements. The remaining points persist and are tracked at the next time step.

Each collection of points is denoted as $\Phi_{t}=$ $\left\{\phi_{t, 1}, \phi_{t, 2}, \cdots, \phi_{t, n}\right\}$, where $t$ indicates the current time step in frames. The superscript indicates the source of the collection of points. The output of the three measurement sources are denoted as $\Phi_{t}^{1}, \Phi_{t}^{2}, \Phi_{t}^{3}$. The output of GFP and Recursive-RANSAC are denoted as $\Phi_{t}^{F}$ and $\Phi_{t}^{R}$ respectively.

The combination and filtering of measurement sources can be shown using set notation. The collection of points resulting from GFP is

$$
\Phi_{t}^{F}=\operatorname{GFP}\left(\Phi_{t}^{1} \bigcup \Phi_{t}^{2} \bigcup \Phi_{t}^{3}\right)
$$

The collection of points resulting from RecursiveRANSAC is

$$
\Phi_{t}^{R}=R R\left(\Phi_{t}^{F}\right)
$$

where RR denotes Recursive-RANSAC. An important characteristic of GFP and R-RANSAC is that neither perform any kind of averaging; they only accept or reject points. Specifically, the collections of points are subsets of each other:

$$
\Phi_{t}^{R} \subseteq \Phi_{t}^{F} \subseteq\left(\Phi_{t}^{1} \bigcup \Phi_{t}^{2} \bigcup \Phi_{t}^{3}\right)
$$

The output of the multi-target tracker is track estimates with position and velocity.

\section{RESULTS}

The complete tracker was tested on multiple video sequences. However, for brevity only results on one of the video sequences are included in this paper. The video sequence is from a hand-held moving camera with significant rotation but almost no translation. This motion is characteristic of UAV motion at high altitudes since the translational component is small in comparison to the altitude of the 
(a)

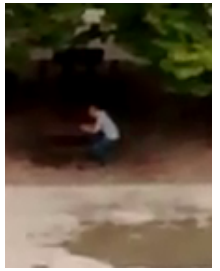

(b)

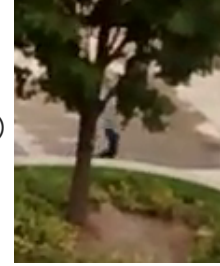

(c)

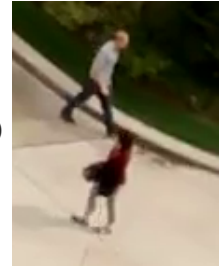

Fig. 8. Challenges in the video sequence. (a) The object is stopped from frame 150 to frame 750 . (b) The object is briefly occluded near frame 1300. (c) The object passes near another target at frame 1580 .

UAV. It is also similar to UAV motion at low altitudes in planar scenes, from the standpoint of the accuracy of the homography transformation. Figure 8 shows the challenges in the video sequence.

The results of the tracker are shown in Figure 9 Tracking using only motion detection is not able to track targets once they stop. As a result the target is lost and later a new track is initialized when the person resumes walking.

Tracking using motion detection and optical flow is able to track stopped targets. However, without a ghost track reduction method, the track is lost when the pedestrian walks behind a tree (near frame 1300). Occasional tracking errors throughout the video sequence also cause the estimate to lag behind the true trajectory.

Tracking using motion detection, optical flow, and ghost track reduction successfully tracks the target when stopped and does not lose the target during the short occlusion.

Adding feature matching in addition to motion detection, optical flow, and ghost track reduction does not noticeably improve the tracking accuracy. This is likely because motion detection and optical flow combined already do a good job distinguishing between foreground and background points. Feature matching is most helpful when distinguishing between interacting targets. However, the Probabilistic Data Association Filter in our implementation does not use the results of feature matching information to determine track association. This is beyond the scope of this paper and will be implemented in future work.

Figure 10 compares the stopped object tracker with other state-of-the-art trackers. The single object tracker (ECO) performs well, but cannot track multiple objects or automatically initialize tracks. The trackers that rely on object detectors (Deep SORT with Faster R-CNN and YOLO) have poor track continuity because the object detectors struggle to detect small objects in the image.

The computation speed in frames per second of each tracker is shown in Table [1. The video size used in the comparison is $1280 \times 720$ pixels. Computation speeds marked with a star $\left(^{*}\right)$ are taken from the original paper describing each method. In our implementation every 3rd frame is used in the video sequence, which means the algorithm is capable of running in real time from a $30 \mathrm{fps}$ camera. The ECO and YOLO trackers are also capable of running in real time. However, our tracker is the only tracker listed capable of tracking multiple objects in real time without a GPU.

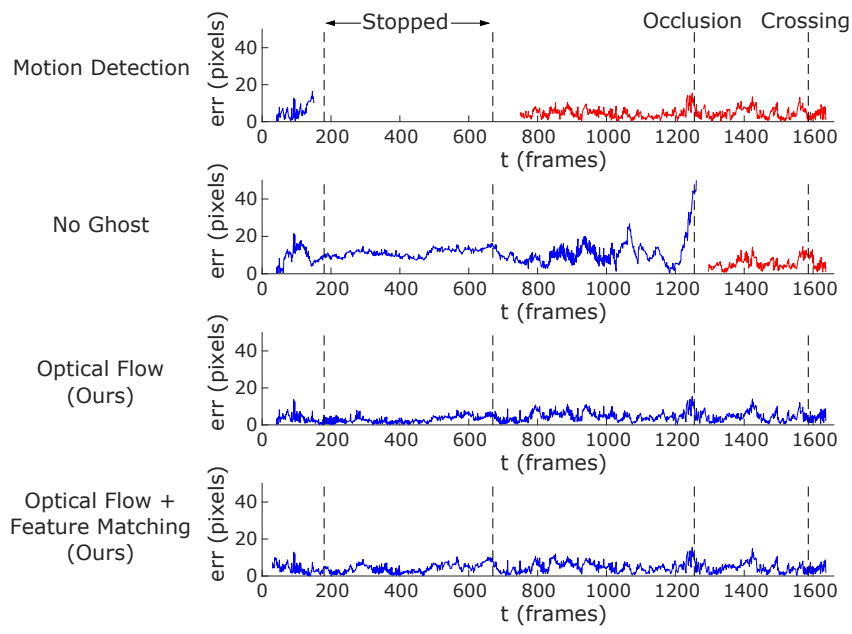

Fig. 9. Tracking accuracy for one of the targets in the video sequence. Each track id is plotted in a separate color. Tracking using only motion detection fails when the object stops. Persistent point tracking without ghost track reduction lags behind and eventually fails at the occlusion. Persistent point tracking using optical flow with ghost track reduction succeeds in tracking the target for the entire video sequence. Adding feature matching to these methods does not noticeably improve the tracking estimate.

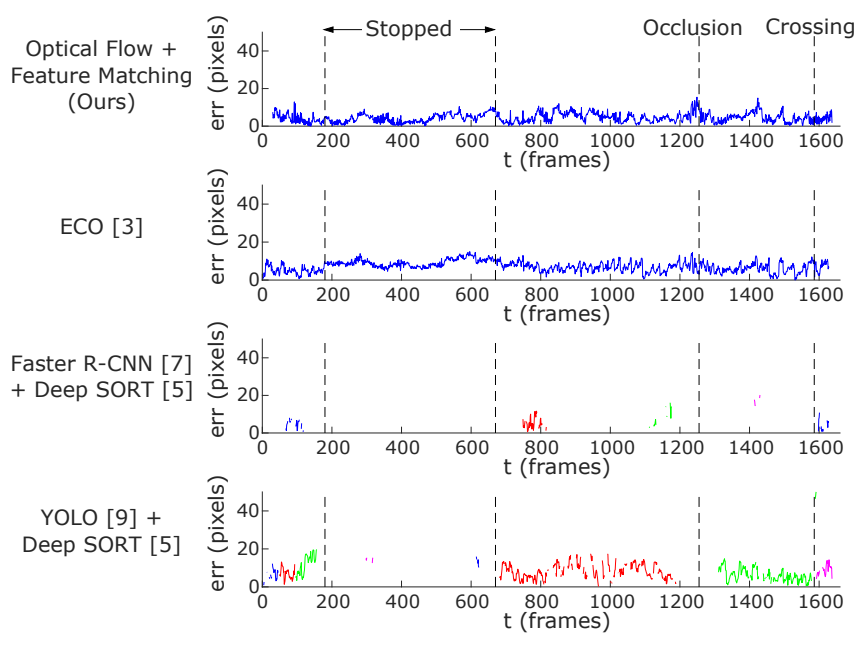

Fig. 10. Comparison with other state-of-the-art trackers for one of the targets in the video sequence. Each track id is plotted in a separate color. Our tracker and the ECO tracker successfully track the target for the entire sequence. Note that the ECO tracker only tracks one target and requires manual track initialization. Tracking using Deep SORT with Faster RCNN detections gives poor track continuity. Using Deep SORT with YOLO instead gives slightly better results, though the track is still quite fragmented.

TABLE I

TRACKER TIMING RESULTS

\begin{tabular}{|c|c|c|}
\hline Tracker & FPS & Hardware \\
\hline \hline Motion detection & 18.5 & i7 CPU \\
\hline No Ghost & 15.6 & i7 CPU \\
\hline Optical Flow (Ours) & 12.2 & i7 CPU \\
\hline Feature matching (Ours) & 10.6 & i7 CPU \\
\hline ECO-HC [3] & $60^{*}$ & K40 GPU \\
\hline Deep SORT [5] with Faster R-CNN (VGG) [7] & $4.6^{*}$ & K40 GPU \\
\hline Deep SORT [5] with YOLOv2 416×416 [9] & $32^{*}$ & Titan X GPU \\
\hline
\end{tabular}




\section{CONCLUSION}

In this work, we have presented a tracker capable of tracking multiple objects in real time from a moving platform. Our tracker combines motion detection, optical flow, and feature matching using a grid-based feature prioritization algorithm that reduces computational complexity and spatial redundancy. Recursive-RANSAC is used to automatically initialize new tracks and reject outlier measurements. Ghost track reduction helps avoid tracking non-existent objects when true objects are no longer visible.

In the future we plan to extend this work to accurately track objects in the presence of parallax. We also plan to use the results of feature matching in the Probabilistic Data Association Filter to better distinguish between interacting targets.

\section{REFERENCES}

[1] H. Nam and B. Han, "Learning multi-domain convolutional neural networks for visual tracking," in Proceedings of the IEEE Conference on Computer Vision and Pattern Recognition, 2016, pp. 4293-4302.

[2] M. Danelljan, A. Robinson, F. S. Khan, and M. Felsberg, "Beyond correlation filters: Learning continuous convolution operators for visual tracking," in European Conference on Computer Vision. Springer, 2016, pp. 472-488.

[3] Danelljan, Martin and Bhat, Goutam and Shahbaz Khan, Fahad and Felsberg, Michael, "ECO: Efficient convolution operators for tracking," in CVPR, 2017.

[4] G. Nebehay and R. Pflugfelder, "Clustering of static-adaptive correspondences for deformable object tracking," in Proceedings of the IEEE Conference on Computer Vision and Pattern Recognition, 2015, pp. 2784-2791.

[5] N. Wojke, A. Bewley, and D. Paulus, "Simple online and realtime tracking with a deep association metric," arXiv preprint arXiv:1703.07402, 2017.

[6] P. F. Felzenszwalb, R. B. Girshick, D. McAllester, and D. Ramanan, "Object detection with discriminatively trained part-based models," IEEE transactions on pattern analysis and machine intelligence, vol. 32, no. 9, pp. 1627-1645, 2010.

[7] S. Ren, K. He, R. Girshick, and J. Sun, "Faster R-CNN: Towards real-time object detection with region proposal networks," IEEE transactions on pattern analysis and machine intelligence, vol. 39, no. 6, pp. 1137-1149, 2017.
[8] A. Milan, L. Leal-Taixé, I. Reid, S. Roth, and K. Schindler, "MOT16: A benchmark for multi-object tracking," arXiv preprint arXiv:1603.00831, 2016.

[9] J. Redmon and A. Farhadi, "YOLO9000: Better, faster, stronger," arXiv preprint arXiv:1612.08242, 2016.

[10] W. Liu, D. Anguelov, D. Erhan, C. Szegedy, S. Reed, C.-Y. Fu, and A. C. Berg, "SSD: Single shot multibox detector," in European conference on computer vision. Springer, 2016, pp. 21-37.

[11] M. Radovic, O. Adarkwa, and Q. Wang, "Object recognition in aerial images using convolutional neural networks," Journal of Imaging, vol. 3, no. 2, p. 21, 2017.

[12] V. Santhaseelan and V. K. Asari, "Moving object detection and tracking in wide area motion imagery," in Wide Area Surveillance. Springer, 2014, pp. 49-70.

[13] V. Reilly, H. Idrees, and M. Shah, "Detection and tracking of large number of targets in wide area surveillance," Computer Vision-ECCV 2010, pp. 186-199, 2010.

[14] P. C. Niedfeldt and R. W. Beard, "Multiple Target Tracking using Recursive RANSAC," in 2014 American Control Conference, June 2014, pp. 3393-3398.

[15] P. C. Defranco, "Detecting and tracking moving objects from a small unmanned air vehicle," Master's thesis, Brigham Young University, 2015. [Online]. Available: http://scholarsarchive.byu.edu/etd/5311/

[16] M. Calonder, V. Lepetit, C. Strecha, and P. Fua, "BRIEF: Binary robus independent elementary features," Computer Vision-ECCV 2010, pp. 778-792, 2010.

[17] S. Baker and I. Matthews, "Lucas-Kanade 20 years on: A unifying framework," International Journal of Computer Vision, vol. 56, no. 3 , pp. 221-255, 2004

[18] J. Shi et al., "Good features to track," in Proceedings of IEEE Conference on Computer Vision and Pattern Recognition CVPR-94. IEEE, 1994, pp. 593-600.

[19] J. Kang, I. Cohen, G. Medioni, and C. Yuan, "Detection and tracking of moving objects from a moving platform in presence of strong parallax," in Computer Vision, 2005. ICCV 2005. Tenth IEEE International Conference on, vol. 1. IEEE, 2005, pp. 10-17.

[20] Y. Sheikh, O. Javed, and T. Kanade, "Background subtraction for freely moving cameras," in Computer Vision, 2009 IEEE 12th International Conference on. IEEE, 2009, pp. 1219-1225.

[21] A. Elqursh and A. Elgammal, "Online moving camera background subtraction," in European Conference on Computer Vision. Springer, 2012, pp. 228-241.

[22] Y. Bar-Shalom, F. Daum, and J. Huang, "The probabilistic data association filter," IEEE Control Systems, vol. 29, no. 6, 2009.

[23] "Opencv 3.1: Open source computer vision library," https://github. com/opencv/opencv/releases/tag/3.1.0 2015.

[24] Z. Kalal, K. Mikolajczyk, and J. Matas, "Forward-backward error: Automatic detection of tracking failures," in Pattern recognition (ICPR), 2010 20th international conference on. IEEE, 2010, pp. 2756-2759. 